\title{
Valor predictivo de progresión de la muscularis mucosae y del porcentaje de células G3 en tumores vesicales T1G3
}

\author{
J.A. Queipo Zaragozá, J.L. Ruiz Cerdá, L.A. Rubio Martínez*, F. Vera Sempere*, A. Budía Alba, \\ J.F. Jiménez Cruz
}

Servicio de Urología, Servicio de Anatomía Patológica*, Hospital Universitario La Fe. Valencia.

Actas Urol Esp 2005; 29 (5): 439-444

RESUMEN

VALOR PREDICTIVO DE PROGRESIÓN DE LA MUSCULARIS MUCOSAE Y DEL PORCENTAGE DE CÉLULAS G3 EN TUMORES VESICALES T1G3

Introducción y Objetivos: El tumor vesical T1G3 constituye la frontera entre el "tumor superficial" y el "tumor infiltrante". Algunos de estos tumores no responden a BCG y progresan, con cistectomías que presentan pobres resultados, pacientes que se beneficiarian de un tratamiento precoz mas agresivo si pudiéramos identificarlos en una etapa preinvasiva. Nuevos factores predictivos intentan seleccionar estos tumores, siendo escasos los trabajos que consideran un estudio anatomopatológico mas minucioso (subestadiaje del T1 en Tla y Tlb y porcentaje de células G3 presentes en el tumor). Nuestro objetivo es analizar el valor de estas consideraciones anatomo-patológicas como factores predictivos de progresión.

Material y Métodos: Estudio retrospectivo de una serie de 91 pacientes afectos de tumor vesical T1G3 sometidos a tratamiento inicial mediante RTU e instilaciones de BCG. Analizamos 12 variables; entre los nuevos factores predictivos: el nivel de invasión respecto de la muscularis mucosae y el porcentaje de células G3. Mediante análisis de regresión logística establecemos las variables pronósticas independientes para progresión tumoral.

Resultados: Un total de 31 pacientes presentaron infiltración del detrusor, falleciendo 17 de causa tumoral, tras un tiempo medio de seguimiento de 57,8 $\pm 28,2$ meses. En 8 casos (9\%) no se pudo determinar el subestadiaje. La tasa de progresión para tumores Tla fue del 20\% (8/40) y para T1b 53\% (23/43). Presentaron valor predictivo independiente de progresión la multiplicidad (odds: 7,26), el tamaño (odds: 2,14), la presencia de Cis (odds: 1,42) y el subestadiaje (odds: 6,81).

Conclusión: El subestadiaje es un factor predictivo de progresión clínicamente útil en tumores vesicales T1G3, por lo que consideramos fundamental su introducción en la práctica clínica habitual.

Palabras clave: T1g3. Muscularis Mucosae. Progresión.

\section{ABSTRACT}

PREDICTIVE VALUE OF PROGRESSION OF MUSCULARIS MUCOSAE AND PERCENTAGE G3 CELLS IN VESICAL T1G3 TUMOURS

Introduction and objective: Vesical tumor T1G3 constitutes the border between the superficial tumor and the infiltrante tumor. Some of these tumors do not respond to BCG and progress, with cystectomy that present poor results, patients who would benefit from a precocious and aggressive treatment if we could identify them in an preinvasive stage. New predictive factors try to select to these tumors, being little the works that consider anatomo-pathological meticulous study (substanding of the T1 in T1a and T1b and percentage of present G3 cells in the tumor). Our objective is to analyze the value of these anatomo-pathological considerations like predictive factors of progression.

Material and methods: Retrospective study of a series of 91 patient affection of vesical tumor T1G3 with initial treatment by means of RTU and BCG. We analyzed 12 variables. The new predictive factors: the level of invasion respect to muscularis mucosae and the percentage of G3 cells. By means of logistic regression analisys we establish the independent pronostic factors for tumoral progression.

Results: A total of 31 patients presented infiltration of detrusor, passing away 17 of tumoral cause, after an average time of pursuit of $57.8 \pm 28.2$ months. In 8 cases $(9 \%)$ the substanding could not be determined. The rate of progression for Tla tumors was of $20 \%$ (8/40) and for T1b 53\% (23/43). Presented independent predictive value of progression the multiplicity (odds: 7,26 ), the size (odds: 2,14), the presence of Cis (odds: 1,42) and the subestanding (odds: 6,81).

Conclusion: The substanding is a predictive factor of progression clinically useful in vesical tumors T1G3, reason why we considered habitual clinical introduction.

Keywords: T1G3. Muscularis Mucosae. Progression. 
$\mathrm{L}$ os tumores vesicales T1G3 constituyen el grupo de tumores superficiales con mayor riesgo de progresión, configurando el límite o frontera entre el tumor "superficial" y el "invasivo”. Un porcentaje importante de pacientes no responden a BCG y progresan, con cistectomías que presentan pobres resultados, pacientes que se beneficiarian de una cistectomía precoz o una pauta de quimio-radioterapia si pudiéramos identificarlos en una etapa preinvasiva. Con las pautas actuales de tratamiento, a 10 años la tasa de exitus llega al $38 \%{ }^{1}$. Por tanto, los factores pronósticos clásicos no han demostrado ser suficientes para identificar aquellos tumores que van a progresar, antes de que ello ocurra. Por ello se investigan nuevos factores predictivos que aporten información añadida a los factores ya conocidos, independiente, y por tanto, clínicamente útil.

Nuestro objetivo es determinar el valor predictivo de progresión del subestadiaje del T1, fundamentalmente en base a la identificación de la muscularis mucosae. Además analizamos el porcentaje de células G3 presentes en el tumor, ya que consideramos diferentes tumores aquellos con abundantes células G3 de los que presentan escasas células G3. Una vez analizados estos factores individualmente, hemos seleccionado aquellos con valor predictivo independiente.

\section{MATERIAL Y MÉTODOS}

Realizamos un estudio retrospectivo sobre una serie inicial de 91 pacientes afectos de tumor vesical T1G3, diagnosticados y tratados entre los años 1986 y 2003 en el Hospital Universitario La Fe de Valencia. Todos los pacientes fueron sometidos inicialmente a resección transuretral completa (RTU) + instilaciones intravesicales de BCG (81 mg cepa Connaught, instilación semanal durante 6 semanas), con un seguimiento minimo de 3 años.

Analizamos un total de 12 variables. Como factores pronósticos clásicos: edad, sexo, tabaquismo, número de tumores, tamaño tumoral, morfología (sésil versus pediculado), consistencia (sólido versus no sólido), localización, presencia o no de ureterohidronefrosis y carcinoma in situ asociado. Entre los nuevos factores pronósticos se determinó el nivel de invasión de la submuco- sa, distinguiendo entre tumores T1a y T1b si el tumor alcanzaba o no el plano de la muscularis mucosae. En los casos en que ésta estructura no se pudo identificar utilizamos como referencia de la misma la presencia de grandes vasos arteriolares que transcurren paralelos a la superficie mucosa. Determinamos el porcentaje de células G3 presentes en el tumor, obteniendo una media de $27,1 \pm 15,7 \%$ y mediana: $20 \%$, estableciendo como punto de corte el de $20 \%$ de células G3, pues dicho porcentaje era el punto de corte que mostraba mejor las diferencias de comportamiento, significativas en el análisis univariante. Establecimos así dos grupos de tumores: grupo A si contienen < 20\% células G3 y grupo B si $\geq$ $20 \%$.

Utilizamos las muestras de RTU contenidas en bloques de parafina. Tras realizar cortes finos de 5 micras de grosor utilizando un microtomo de rotación con control digital (Micron $H-M$ 340), se tiñeron las laminillas con la técnica hematoxilina-eosina, seleccionando el material biópsico idóneo para efectuar nuestro estudio. El subestadiaje se estableció, como ya se ha señalado, en base a la invasión por debajo (T1a) (Fig. 1) o más allá (T1b) (Fig. 2) de la "muscularis mucosae".

La valoración de las laminillas fue realizada por 2 observadores y sin conocimiento de la evolución clínica de los pacientes; en aquellos casos en que se obtuvieron resultados contradictorios, se procedió a una reevaluación y se alcanzó un consenso.

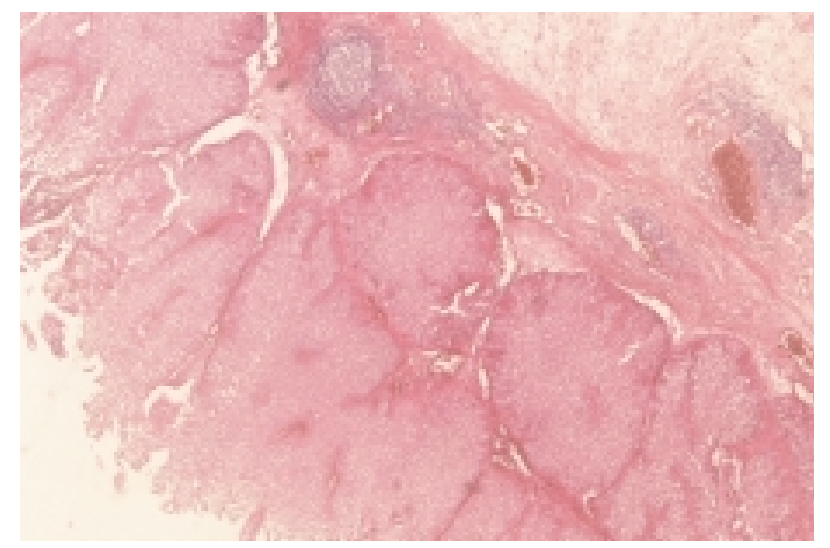

FIGURA 1. Subestadio T1a: Tumor papilar con crecimiento de predominio exofitico, con frente de crecimiento expansivo que afecta a la lámina propia superficial respetando la muscularis mucosae. HE 100x. 


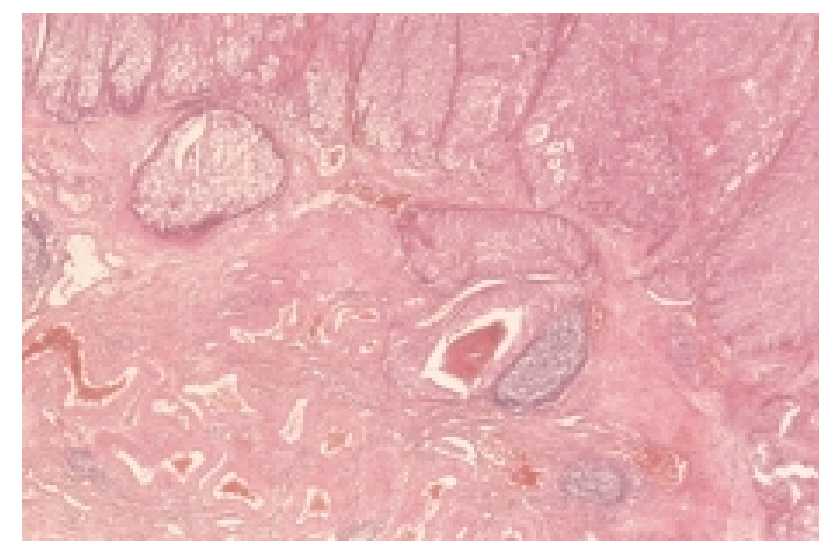

FIGURA 2. Subestadio T1b: Tumor papilar con nidos de infiltración en su base que afectan al espesor de la lámina propia alcanzando de forma focal el plano de la muscularis mucosae, marcada ésta por estructuras vascula res de paredes gruesas. HE 100x.

Para el análisis estadístico se introdujeron las variables en una base de datos, empleando el programa estadístico SPSS para Windows versión 10.0 de SPSS Inc. Todas las pruebas estadísticas se realizaron con una significación de 0,05 y las estimaciones con un intervalo de confianza del 95\%. Las funciones de supervivencia se establecieron por el método de Kaplan-Meier y el test de log-rank. Para determinar los factores pronósticos independientes utilizamos un modelo de regresión logística.

\section{RESULTADOS}

Sólo 83 pacientes fueron considerados en el estudio, al no poder determinar el subestadiaje en 8 de los 91 que constituían la serie inicial. De los 83 pacientes incluidos, 71 fueron varones $(86 \%)$ y 12 mujeres (14\%), con una relación varón/mujer de 6:1. La edad media fue de 68,1 \pm 8,5 años. El tiempo medio de seguimiento ha sido de 57,8 $\pm 28,2$ meses (rango: 13-124), constando actualmente 49 pacientes vivos sin enfermedad, 4 vivos con enfermedad, 13 exitus por causa no tumoral y 17 exitus de causa tumoral. Hubo recidiva en 60 pacientes. Un total de 31 han presentado infiltración del detrusor, 17 de ellos en la primera recidiva; al año de seguimiento el $23 \%$ de los pacientes habían progresado y a los 3 años el 34\%. Los 17 pacientes fallecidos de causa tumoral suponen el 55\% de los tumores que habían progresado y el $28 \%$ de los pacientes con recidiva.
La distribución de casos y tasas de progresión para los factores pronósticos clásicos asociados de forma significativa a progresión tumoral en el análisis univariante se describen en la Tabla 1. Las curvas actuariales pusieron de manifiesto que los pacientes con tumores múltiples, mayores de $3 \mathrm{~cm}$, sólidos, sesiles, con hidronefrosis o con Cis asociado, presentaron un tiempo libre de progresión significativamente menor que aquellos con tumores únicos, menores de $3 \mathrm{~cm}$, no sólidos, pediculados, sin hidronefrosis y sin Cis.

\section{Tabla 1}

Distribución de casos y tasas de progresión para las variables con valor pronóstico significativo en el análisis univariante

\begin{tabular}{lccc}
\hline Variable & Categoría & $\begin{array}{c}\text { No casos/ } \\
\text { Total }\end{array}$ & $\begin{array}{c}\text { Tasa de } \\
\text { progresión (\%) }\end{array}$ \\
\hline № Tumores & Único & $8 / 35$ & 23 \\
& Múltiple & $23 / 48$ & 48 \\
Tamaño & $\leq 3 \mathrm{~cm}$ & $14 / 49$ & 29 \\
& $>3 \mathrm{~cm}$ & $17 / 34$ & 50 \\
Morfologia & Pediculado & $12 / 45$ & 27 \\
& Senil & $19 / 38$ & 50 \\
Consistencia & No sólido & $11 / 46$ & 24 \\
& Sólido & $20 / 37$ & 54 \\
Hidronefrosis & No & $21 / 66$ & 32 \\
\multirow{2}{*}{ Carcinoma in situ } & Si & $10 / 17$ & 59 \\
& No & $19 / 61$ & 31 \\
& Si & $12 / 22$ & 55
\end{tabular}

Un total de 40 pacientes fueron clasificados como Tla (44\%) frente a $43 \mathrm{Tlb}(47 \%)$; en 8 casos (9\%) no se pudo determinar el subestadiaje. La tasa de progresión para Tla fue del $20 \%(8 / 40)$ y para T1b 53\% (23/43). Los pacientes en estadio T1b presentaron un tiempo libre de progresión significativamente menor que los pacientes con estadios Tla $(\mathrm{p}<0,002)$ (Fig. 3). Respecto al contenido en células G3, 43 pacientes $(52 \%)$ se incluyeron en el grupo A y 40 (48\%) en el B. Los tumores con alto porcentaje de células G3 presentaban un tiempo libre de progresión significativamente menor que aquellos con menor porcentaje de células G3 (Fig. 4). Además el análisis de asociación con las demás variables demostró que los tumores con mayor porcentaje de células G3 presentaban una proporción mayor de estadios T1b. 


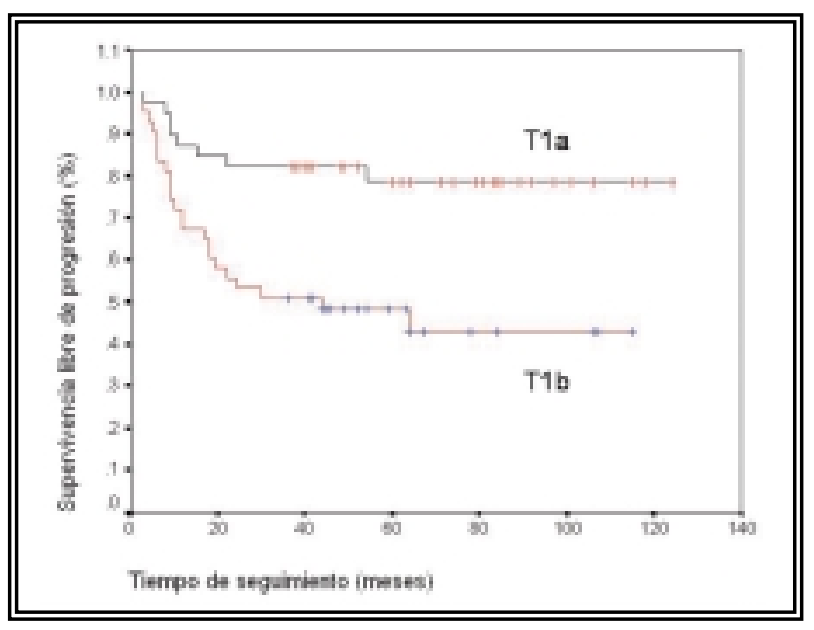

FIGURA 3. Probabilidad de progresión en función del microestadiaje.

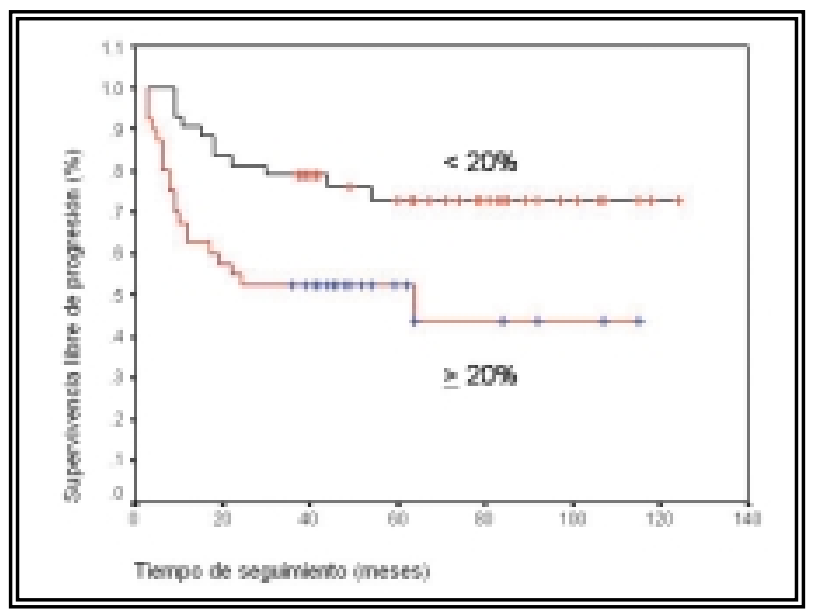

Figura 4. Probabilidad de progresión en función de la proporción de células G3.

El análisis multivariante, a partir de un modelo de regresión logística, seleccionó 4 factores como predictores independientes de progresión a enfermedad invasiva: numero de tumores-multiplicidad (odds: 7,26), tamaño tumoral mayor de 3 cm (odds: 2,14), presencia de Cis (odds: 1,42) y subestadiaje T1b (odds: 6,81) (Tabla 2).

\section{DISCUSION}

\section{Subestadiaje del T1}

El subestadiaje considera la muscularis mucosae como una verdadera frontera en la evolución del tumor, estableciendo diferencias pronósticas en base a su invasión o no. Actualmente no está incluido en el TNM, ya que la muscularis mucosae es una estructura anatómica inconstante; sin embargo, los informes histopatológicos cada vez mas distinguen hoy la presencia de esta estructura. Además, se hace necesario demostrar su valor predictivo independiente, clínicamente útil.

A partir de la identificación de la muscularis mucosae diversos trabajos consiguen subestadiar el tumor en poco mas del 55\% de piezas de $\mathrm{RTU}^{2,3}$. Por ello, consideran que es necesario introducir criterios adicionales de subestadificación. Estimando como referencia de la muscularis mucosae la identificación de las arterias de la lámina propia (vasos de gran calibre y de trayecto paralelo a la superficie mucosa), algunos autores consiguen determinar el nivel de invasión en mas del $90 \%$ de casos $^{4,5}$. Bernardini S y cols. ${ }^{6}$ aunque sólo identifican la muscularis mucosae en el $70 \%$ de tumores, logran subestadiar el $100 \%$ de ellos. Otros autores ${ }^{7}$ establecen la subestadificación en función de la profundidad de invasión de la lámina propia, medida con un micrómetro.

En nuestro trabajo conseguimos identificarla en 76 pacientes $(84 \%$ del total de la serie), presentándose ésta, mayoritariamente, con un patrón focal; pero, utilizando como referencia las arterias de la lámina propia, logramos determinar el nivel de invasión en un total de 83 pacientes (91\% de la serie).

Respecto a su valor como factor pronóstico la mayoría de estudios han analizado tumores $\mathrm{T} 1$, sin referirse a un grado tumoral concreto. Ya a principios de los años 90 diversos autores obser-

Tabla 2

Modelo de regresión logística. Factores pronósticos con valor predictivo independiente de progresión tumoral

\begin{tabular}{lcccccc}
\hline & Coeficiente B & $\begin{array}{c}\text { Error } \\
\text { Estandar }\end{array}$ & Wald X2 & $\mathbf{p}$ & $\begin{array}{c}\text { Odds } \\
\text { Ratio }\end{array}$ & $\begin{array}{c}\text { Intervalo de } \\
\text { Confianza (95\%) }\end{array}$ \\
\hline Constante & $-9,85$ & 2,41 & 17,20 & $<0,001$ & & \\
Multiplicidad & 1,72 & 0,69 & 7,53 & 0,0073 & 7,26 & $1,70-30,78$ \\
Tamaño (3cm) & 0,76 & 0,58 & 1,47 & 0,2281 & 2,14 & $0,61-6,84$ \\
Cis & 0,30 & 0,61 & 0,33 & 0,5745 & 1,42 & $0,40-5,07$ \\
$\mu$ Mestadiaje & 1,74 & 0,66 & 8,35 & 0,0031 & 6,81 & $1,81-24,32$ \\
\hline
\end{tabular}


vaban diferentes tasas de supervivencia en función del subestadiaje ${ }^{8,9}$. En trabajos más recientes, que consideran el análisis multivariante ${ }^{2-4,6}$, la muscularis mucosae aparece como factor pronóstico independiente tanto de recurrencia como de progresión y supervivencia. Sin embargo, Platz CE y cols. no observan diferencias de supervivencia a 10 años ${ }^{10}$. En el grupo específico de tumores T1G3, Holmang y cols ${ }^{5}$, encuentran tasas de progresión mas altas para Tlb que para Tla (58\% vs $36 \%$ ) y el doble de riesgo de morir de cáncer vesical ( $45 \%$ vs $23 \%)$. Utilizando como criterio de subestadificación la profundidad de invasión medida en micras, Chen y cols., también obtienen un valor predictivo independiente de progresión a 5 años, con unas tasas libres de progresión del 67\% y $93 \%$ en tumores con invasión mayor ó menor de 1,5 $\mathrm{mm}$ respectivamente ${ }^{7}$.

$\mathrm{El}$ análisis de regresión logística en nuestra serie mostró que este factor presenta valor predictivo independiente de progresión a $\mathrm{T} 2$, con una odds ratio muy elevada, resultados similares a los obtenidos por Bernardini S y cols ${ }^{6}$.

\section{Porcentaje de células G3}

En el año 1998 se presentaron los criterios de consenso de la OMS (Organización Mundial de la Salud) y de la SIPU (Sociedad Internacional de Patólogos Urológicos) con respecto a la gradación de los tumores vesicales ${ }^{11}$. Pero hasta la fecha, la antigua clasificación de la OMS de $1973^{12}$, sigue siendo la norma, estando pendiente entre los patólogos la difusión de la nueva clasificación. La gradación clásica de Mostofi (OMS 1973) establece, además del papiloma (grado 0), el carcinoma transicional grado I, el carcinoma transicional grado II y el carcinoma transicional grado III, dependiendo de los parámetros morfológicos de su celularidad tumoral: pérdida de polaridad y diferenciación, pleomorfismo nuclear (patrón cromatínico), actividad mitótica y presencia de células gigantes ${ }^{12}$, determinándose la gradación en función del peor grado presente ${ }^{13}$. En la clasificación propuesta por el grupo de consenso de la OMS/SIPU de 1998 se introdujo el término de carcinoma de alto grado en sustitución del carcinoma grado 3 .

Las características morfológicas de las células tumorales continúan siendo, a pesar del desarro- llo de las más modernas técnicas de citogenética, un factor fundamental en el manejo del cáncer vesical, aunque, con una constante reevaluación morfológica, en un intento de mejorar su valor predictivo. Los intentos por eliminar el componente subjetivo del patólogo en su interpretación del grado han hecho que se hayan investigado diferentes procedimientos como la morfometría nuclear, la determinación de regiones organizadoras de nucléolos con tinción de plata y técnicas citogenéticas como el estudio de la ploidía, que aunque han tenido éxito demostrando un valor pronóstico significativo, no se utilizan de forma sistemática $^{14,15}$.

Es bien conocido que los carcinomas grado 2 presentan un comportamiento biológico heterogéneo, lo que ha hecho que diversos autores hayan intentado subdividirlo, en busca de una mayor correlación pronóstica. Así, Carbin E y cols. ${ }^{16}$ en 1991, establecieron dos subgrupos pronósticos, G2a y G2b, observando que los tumores G2a se aproximaban en su evolución a los carcinomas grado 1 (supervivencia a 5 años del 92\%), y los G2b se comportaban de una forma más próxima a los G3 (supervivencia a 5 años del 43\%). Pero esta subclasificación del grado 2 no se ha popularizado.

Los tumores grado 3 constituyen las formas histológicas más agresivas y de peor pronóstico a largo plazo. Uno de los planteamientos en este trabajo ha sido establecer dos subgrupos pronósticos en estos tumores T1G3, en función de la proporción de células de alto grado presentes, pues consideramos que no es lo mismo un tumor que presenta una histología con abundantes células G3, que aquel que también es clasificado como de alto grado por el hecho de tener algunas células G3. Hemos revisado cuidadosamente la literatura y no hemos encontrando consideraciones al respecto. Nuestros resultados ponen en evidencia la mayor agresividad en función del porcentaje de células G3 pero sin valor clínicamente útil frente al resto de factores pronósticos.

\section{CONCLUSIONES}

De entre los nuevos factores predictivos propuestos, sólo presentaría información clínicamente útil la determinación del subestadiaje. Sin embargo, no siempre es posible su determinación, lo que hace que se deban establecer unos 
criterios universales y posteriormente su papel en la predicción de la progresión tumoral. A tenor de nuestros resultados consideramos que la introducción del subestadiaje como factor pronósticos es fundamental para el establecimiento de las estrategias de tratamiento de los pacientes con tumores vesicales pT1G3.

\section{REFERENCIAS}

1. Haukaas S, Daehlin L, Maartmann-Moe H, Ulvik NM. The long-term outcome in patients with superficial transitional cell carcinoma of the bladder: a single-institutional experience. BJU Int 1999; 83:957-963.

2. Angulo JC, Lopez JI, Grignon DJ, Sanchez-Chapado M. Muscularis mucosa differentiates two populations with different prognosis in stage T1 bladder cancer. Urology 1995; 45:47-53.

3. Sozen S, Akbal C, Sokmensuer C, Ekici S, Ozen H. Microstaging of pT1 transitional cell carcinoma of the bladder. Does it really differentiate two populations with different prognoses? (pT1 subcategory). Urol Int 2002; 69:200206.

4. Hermann GG, Horn T, Steven K. The influence of the level of lamina propria invasion and the prevalence of p53 nuclear accumulation on survival in stage T1 transitional cell bladder cancer. J Urol 1998; 159: 91-94.

5. Holmang S, Hedelin H, Anderstrom C, Holmberg E, Johansson SL. The importance of the depth of invasion in stage T1 bladder carcinoma: a prospective cohort study. J Urol 1997; 157:800-803.

6. Bernardini S, Billerey C, Martin M, Adessi GL, Wallerand H, Bittard $\mathrm{H}$. The predictive value of muscularis mucosae invasion and p53 over expression on progression of stage $\mathrm{T} 1$ bladder carcinoma. J Urol 2001; 165: 42-46.

7. Cheng L, Weaver AL, Neumann RM, Scherer BG, Bostwick DG. Substaging of T1 bladder carcinoma based on the depth of invasion as measured by micrometer: a new proposal. Cancer 1999; 86:1035-1043.
8. Younes, M., Sussman, J., True, L.D.: The usefulness of the level of the muscularis mucosae in the staging of invasive transitional cell carcinoma of the urinary bladder. Cancer 1990; 66:543-548.

9. Hasui Y, Osada Y, Kitada S, Nishi S. Significance of invasion to the muscularis mucosae on the progression of superficial bladder cancer. Urology 1994; 43:782-786.

10. Platz CE, Cohen MB, Jones MP, Olson DB, Lynch CF. Is microstaging of early invasive cancer of the urinary bladder possible or useful? Mod Pathol 1996; 9:1035-1039.

11. Epstein JI, Amin MB, Reuter VR, Mostofi FK. The World Health Organization International Society of Urological Pathology consensus classification of urothelial (transitional cell) neoplasms of the urinary bladder. Bladder Consensus Conference Committee. Am J Surg Pathol 1998; 22:1435-1448.

12. Mostofi FK, Swobin LH, Torlini H. Histological Typing of Urinary Bladder Tumours. International Histological Classification of Tumours № 10. Geneve, World Healt Organization, 1973.

13. Bostwick DG, Ramnani D, Cheng L. Diagnosis and grading of bladder cancer and associated lesions. Urol Clin North Am 1999; 26:493-507.

14. Jarkrans T, Vasko J, Bentgsson E y cols. Grading of transitional cell bladder carcinoma by image analysis of histological sections. Anal Cell Pathol 1995; 8135-158.

15. Kruger S, Muller H. Correlation of morphometry, nucleolar organizer regions, proliferating cell nuclear antigen and Ki67 antigen expression with grading and staging in urinary bladder carcinomas. Br J Urol 1995; 75:480-484.

16. Carbin BE, Ekman P, Gustafson H, Christensen NJ, Silfversward C, Sandstedt B. Grading of human urothelial carcinoma based on nuclear atypia and mitotic frequency. II. Prognostic importance. J Urol 1991; 145:972-975.

Dr. J.A. Queipo Zaragozá

Calle Santa Teresa № $4-\mathrm{P}^{\mathrm{a}}$ 8. Puzol

46530 Valencia

(Trabajo recibido el 29 de octubre de 2004) 